\title{
A procedure for in vitro evaluation of the immunosuppressive effect of mesenchymal stem cells on activated $T$ cell proliferation
}

\section{Catalina Marinescu}

Institutul de Biologie si Patologie Celulara Nicolae Simionescu

\section{Bogdan Preda}

Institutul de Biologie si Patologie Celulara Nicolae Simionescu

Alexandrina Burlacu ( $\sim$ sanda.burlacu@icbp.ro)

Institutul de Biologie si Patologie Celulara Nicolae Simionescu https://orcid.org/0000-0001-8811-2098

\section{Methodology}

Keywords: mesenchymal stem cells, T cells, immunosuppression, CFSE, proliferation index, nylon wool, splenocytes, cell culture, flow cytometry

Posted Date: August 26th, 2020

DOI: https://doi.org/10.21203/rs.3.rs-63604/v1

License: (c) (i) This work is licensed under a Creative Commons Attribution 4.0 International License. Read Full License 


\section{Abstract}

Background: Mesenchymal stem/stromal cells (MSC) represent adult cells with multipotent capacity. Besides their capacity to differentiate into multiple lineages in vitro and in vivo, increasing evidence points towards the immunomodulatory capacity of these cells, as an important feature for their therapeutic power. Although not included in the minimal criteria established by the International Society for Cellular Therapy as a defining MSC attribute, demonstration of the immunomodulatory capacity of MSC can be useful for the characterization of these cells before being considered MSC. Here we present a simple and reliable protocol by which the immunosuppressive effect of MSC can be evaluated in vitro. It is based on the measuring of the proliferation of activated T cells cultured in direct contact with irradiated MSC.

Results: Our results showed that MSC have a dose-dependent inhibitory effect on activated T cell proliferation, which can be quantified as a percentage of maximum proliferation. Our data shows that batch-to-batch variability can be determined within one or multiple experiments, by extracting the area under curve of T cell proliferation plotted against the absolute number of MSC in co-culture.

Conclusions: The validation of the immunomodulatory capacity of MSC could be added to the characterization of the cells before being used in various MSC-based approaches to treat immunological diseases. Our results showed that MSC have a dose-dependent inhibitory effect on activated T cell proliferation. The immunosuppressive properties of MSC vary between batches, but not between different passages of the same batch.

\section{Background}

Mesenchymal stem/stromal cells (MSC) represent adult cells with multipotent capacity (1). They were historically isolated from bone marrow; however, their abundance in the adipose tissue had been largely documented thereafter. Besides the two major sources of MSC, a lot of organs have been acknowledged to host such cells, which make their identity sometimes disputable (2-4).

According to International Society for Cellular Therapy (ISCT), MSC are defined as spindle shaped plasticadherent cells, which are negative for hematopoietic markers (CD45, CD14, CD11), positive for several stromal markers (CD105, Sca-1, CD29, CD44) and have the capacity to differentiate into osteoblasts, chondrocytes and adipocytes, when cultured under appropriate conditions (5). Increasing evidence points towards the immunomodulatory capacity of these cells, as an important feature that can explain the therapeutic power of these cells (6-8). Based on this quality, MSC are increasingly used in clinic in bone marrow transplants to cure important diseases with immune component, such as autoimmune diseases, graft-versus-host disease and allograft rejection $(9,10)$.

Although not included in the minimal criteria established by ISCT as a defining MSC characteristic, the immunosuppressive potency of the cells can help characterize these cells before being considered MSC. 
This functional properties of MSC should be quantified by standardized immune assays with purified cells as responders, as already proposed several years ago (11).

Here we present a standardized and reliable protocol by which the immunosuppressive potency of MSC can be certified in vitro. It is based on the assessment of the proliferation index of activated T cells in direct interaction with irradiated MSC in culture.

\section{Methods}

\section{MSC isolation and characterization.}

MSC were isolated from bone marrow aspirate of male 6-week old C57BI/6 mice as previously described (12). Cells were used between 6 th and 12th passages. MSC were routinely characterized for the presence of specific surface markers (by flow cytometry) and for the potential to generate adipocytes, osteoblasts and chondrocytes, when cultured under appropriate conditions (Fig. 1). Flow cytometry analysis demonstrated the absence of the hematopoietic cell marker CD45 and the presence of surface antigens usually associated to MSC (Sca-1, CD105, CD29, and CD90). Adipocytes, osteoblasts and chondrocytes were evidenced by Oil Red $\mathrm{O}$ staining of lipid droplets (Fig. 1a), von Kossa staining of calcium deposits (Fig. 1b), and Alcian Blue staining of acid mucopolysaccharides (Fig. 1c), respectively.

\section{Assessment of the immunosuppressive potential of MSC.}

The procedure has three major steps, which are to be performed in different days:

Step 1 (Day 0; 2 hours): Mitotic inactivation of MSC followed by seeding on tissue-culture wells. Around $1.5 \times 10^{\wedge} 5$ cells are enough for the procedure. This step is performed one day before the interaction of MSC with T cells.

Step 2 (Day $1 ; \sim 6$ hours): Isolation of splenic T cells and fluorescent labeling with Carboxy-fluorescein succinimidyl ester (CFSE) before the addition onto MSC culture in the presence of CD3/CD28 activation beads.

Step 3 (Day 4, 4 hours, including flow cytometry analysis): Assessment of the proliferation index of T cells after three days in culture, based on CFSE dye dilution assay.

\section{MSC irradiation.}

MSC were detached with $0.25 \%$ trypsin and re-suspended at a density of $10^{\wedge} 5$ cells $/ \mathrm{ml}$ in MSC complete medium (DMEM low glucose, supplemented with 10\% MSC qualified FBS, in the presence of $1 \%$ 
antibiotic-antimycotic solution). Cell irradiation was performed with a RS-2000 X-ray irradiator at 30 Gy dose, in 1.5-ml microcentrifuge tube. We routinely irradiated the tubes in the middle of the third shelf (for an evenly radiation distribution) for 16 minutes and 36 seconds. After irradiation, cells were seeded in 96well plate wells, using serial dilutions ranging from 10.000 to 625 cells/well. Technological triplicates were used for each MSC dilution. The irradiated cells were placed in the incubator under $37^{\circ} \mathrm{C}$ and $5 \%$ $\mathrm{CO}_{2}$ atmosphere and left overnight before interaction with $\mathrm{T}$ cells.

\section{Isolation of mouse splenocytes.}

Mice were sacrificed by cervical dislocation and prepared for dissection by disinfecting the fur and skin with $70 \%$ alcohol. The peritoneum was exposed through a midline incision of the skin. The peritoneal wall was cut along the linea alba and then laterally under the ribcage, towards the left side of the mouse. The bowels were gently grabbed and pulled aside to expose the spleen, which was detached (by cutting the connections with other tissues) and introduced into a 15-ml tube containing $2 \mathrm{ml}$ ice-cold PBS/FBS (PBS supplemented with $2 \%$ FBS). Under the laminar flow hood, the tube content (spleen and PBS/FBS) was transferred into a $70-\mu \mathrm{m}$ cell strainer adapted on the top of a 50-ml tube. The spleen was gently triturated (without cutting in pieces), using the thumb head of a 5 -ml syringe plunger, while adding $10 \mathrm{ml}$ of PBS/FBS to wash the strainer. The cell suspension was centrifuged at $400 \mathrm{Xg}, 4 \llbracket \mathrm{C}$, for 10 minutes, then the cell pellet was resuspended in $5 \mathrm{ml}$ ice-cold red blood cell lysis buffer and incubated at $24^{\circ} \mathrm{C}$ for 4 minutes. The lysis was stopped by adding $10 \mathrm{ml}$ ice-cold PBS/FBS and centrifugation at 400Xg, 4区C, 10 minutes. Cells were resuspended in $2 \mathrm{ml}$ complete RPMI medium (RPMI ATCC-formula, supplemented with $10 \%$ FBS and $50 \mu \mathrm{M} \beta$-Mercapto-ethanol), counted and used in T cell enrichment step. An aliquot of around $20 \mu \mathrm{l}\left(\sim 10^{\wedge} 6\right.$ cells) was saved for flow-cytometry analysis of splenocytes.

\section{T cell enrichment from splenocytes using the nylon wool column.}

The protocol described by Hathcock, with a few minor changes, was used with good results (13). The nylon wool column was prepared in advance by inserting $1 \mathrm{~g}$ of nylon wool into a 10-ml sterile syringe and autoclaving at $110 \mathbb{} \mathrm{C}$ for 15 minutes. Briefly, $T$ cell enrichment followed these steps: 1 ) the column was placed on the stand and a 3-way stopcock with a $19 \mathrm{G}$ needle was attached to the column; 2) with the stopcock in open position, the column was washed 3 times with $8 \mathrm{ml}$ warm RPMl complete medium.

During the first wash, the wool was firmly pressed with a 10-ml sterile serological pipette to remove the air bubbles; 3 ) the stopcock was closed, then $2 \mathrm{ml}$ warm complete RPMI medium was added to the column and the column was allowed to equilibrate in the incubator for at least 45 minutes; 4 ) the column was moved back in the cell culture hood, the stopcock was opened and the column was let to drain completely; 5) the 2-ml splenocyte suspension was carefully layered on the column surface (not on the wall of the syringe) and the suspension was allowed to drain completely; 6) the column was washed with $0.5 \mathrm{ml}$ warm complete RPMI medium to ensure that all cells had penetrated the wool; 7) the stopcock was 
closed, and $2 \mathrm{ml}$ warm complete RPMI medium was added onto the column before being placed in the incubator for another 45 minutes; 8 ) the column was removed from the incubator, the $19 \mathrm{G}$ needle was carefully replaced with a $23 \mathrm{G}$ needle, the stopcock was opened and T cells were eluted by washing 4 times with $10 \mathrm{ml}$ warm complete RPMI medium; 9$)$ the eluate $(\sim 40 \mathrm{ml})$ was centrifuged at $400 \mathrm{Xg}, 24^{\circ} \mathrm{C}$, 10 minutes, and then the cells were resuspended in $1 \mathrm{ml}$ warm medium. An aliquot of $50 \mu \mathrm{l}$ was saved for counting (with an intermediate dilution of $1 / 50$ ) and flow-cytometry analysis. The remaining cells were washed with $10 \mathrm{ml} \mathrm{PBS} / \mathrm{FBS}$ and centrifuged at $400 \mathrm{Xg}, 24^{\circ} \mathrm{C}$ for 10 minutes. At the end, the cells were resuspended in 950 ul PBS + 1\% FBS and transferred in a 15-ml conical tube.

\section{T cell labeling with CFSE.}

Uniform labeling of T cells is important for accurate determination of cell proliferation. Our protocol followed the next steps: 1) First, a vortex was brought in the hood and $50 \mu$ CFSE $100 \mu \mathrm{M}$ was freshly prepared in PBS with 1\% FBS (from a concentrated stock solution); 2) While keeping the tube with $950 \mu \mathrm{l}$ cell suspension in a $45 \otimes$ position, the $50-\mu l$ CFSE solution was carefully placed as a stable drop on the upper part of the wall inside the 15-ml tube, making sure that the cell suspension and CFSE solution did not make contact; 3 ) the vortex was turned on, then the lid on the tube was closed and immediately turned upside down and vortex for 1-2 seconds to ensure the rapid and homogenous dispersal of CFSE solution (the final concentration of CFSE was $5 \mu \mathrm{M}$ ); 4) the tube was then incubated at $24{ }^{\circ} \mathrm{C}$ in the dark for 10 minutes; 5) the 15-ml tube was filled up with complete RPMI medium and centrifuged at $400 \mathrm{Xg}$, $4{ }^{\circ} \mathrm{C}, 10$ minutes; 6 ) the supernatant was decanted and the wash procedure was repeated for another 2 times with $10 \mathrm{ml}$ complete RPMI medium; 7) the cell pellet was resuspended in complete RPMI medium at a cell density of $10^{\wedge} 6$ cells $/ \mathrm{ml}$ for plating. An aliquot of $200 \mu \mathrm{l}$ (around $2 \times 10^{\wedge} 5$ cells) was saved for flow-cytometry validation of the homogenous staining.

\section{Co-culture of T cells with the irradiated MSC.}

The immunosuppressive effect of MSC was evaluated by culturing T cells with irradiated MSC in activating conditions. Different MSC: T cell ratios, within the range 1:10 to 1:160, were obtained by serial dilutions of MSC suspension, while keeping the number of T cells constant. The greatest number of MSC per well $\left(10^{4}\right.$ cells/well) was calculated so that to obtain a confluent layer of inactivated cells. Activation of $T$ cells was done by mixing T cells with anti-CD3/CD28 microbeads in a 1:1 ratio (bead: $T$ cell) in RPMI complete medium. Control wells important in this step: irradiated MSC only (to ensure that after 3 days in culture, irradiated MSC are healthy); activated T cells cultured without MSC (to determine the maximum level of T cell proliferation in culture); T cells cultured in the absence of anti-CD3/CD28 beads (to establish the fluorescence median value of CFSE after three days in culture for accurate assessment of the proliferative index).

\section{Flow-cytometry analysis.}


After 3-days of co-culture of T cells with MSC, all cells and beads in the wells were collected for flow cytometry analysis. To achieve this, the following steps were performed: 1) the supernatant of each well was collected in separate $1.5-\mathrm{ml}$ microcentrifuge tubes; 2 ) the wells were washed with $100 \mu \mathrm{l} \mathrm{PBS} /$ well with recovering of the washed volume in the corresponding tube; 3 ) the cells were trypsinized by incubating each well with $30 \mu$ Trypsin-EDTA solution (0.25\% Trypsin mixed with $0.2 \mathrm{~g} / \mathrm{I} \mathrm{EDTA}-4 \mathrm{Na}$ in Hank's Balanced Salt Solution) for two minutes, followed by trypsin inactivation with $100 \mu$ complete RPMI medium and collection of whole volume in the corresponding tube; 4) each well was washed with an additional $100 \mu \mathrm{l}$ PBS, and the washed volume was saved in the same corresponding tubes (the final volume in each tube was around $450 \mu \mathrm{l}) ; 5$ ) the tubes containing beads were placed in the magnet for 2 minutes, and the cell-containing medium was recovered in fresh tubes; 6 ) the beads were washed with $500 \mu \mathrm{l} \mathrm{PBS} /$ FBS by pipetting and re-placing the tubes into the magnet for another 2 minutes; the supernatant was recovered in the corresponding fresh tube (the total volume of cells was around $1 \mathrm{ml} /$ tube); 7) the cells were centrifuged at $300 \mathrm{Xg}, 4^{\circ} \mathrm{C}, 5$ minutes and resuspended in $250 \mathrm{ul} \mathrm{PBS} / \mathrm{FBS}$;

8) $2 \mu \mathrm{g} / \mathrm{ml}$ propidium iodide was added to each sample and cells were analyzed using a Beckman Coulter CytoFlex flow cytometer.

\section{Results}

\section{Characterization of T cells.}

Schematic illustration of the procedures used for splenocyte isolation and T cell enrichment on nylon wool is given in Fig. 2a, together with flow cytometry characterization of cells obtained after each step (Fig. $2 \mathrm{~b}$ ). Around $10^{\wedge} 8$ splenocytes are obtained from one spleen of adult $\mathrm{C} 57 \mathrm{BI} / 6 \mathrm{~J}$ mouse, with a B cell: T cell ratio of more than 2:1 and a ratio of $1.5 \mathrm{CD} 8$ cells to $1 \mathrm{CD} 4$ cell within $\mathrm{T}$ cell sub-population. Our results showed that the percentage of T cells in T cell-enriched population was more than 3-fold increased as compared to that in the splenocyte population. The nylon wool treatment did not importantly alter the ratio between $\mathrm{CD} 4^{+}$and $\mathrm{CD} 8^{+}$cells in $\mathrm{T}$ cell population, as compared to splenocyte population. CFSE staining produced a uniformly stained population, which was demonstrated by the sharp peak on FITC fluorescence channel. Importantly, CFSE-labeled T cells at time $t=0$ is not to be used as the negative control for T cell proliferation, because part of the incorporated CFSE is lost during the 3day culture and, consequently, the fluorescence of CFSE-labeled resting T cells after 3 days in culture is one order of magnitude lower than the fluorescence at time $t=0$.

\section{Co-culture of CFSE-labeled T cells with the irradiated MSC.}

The experimental set-up and the design of the 96-well plate wells needed for evaluation of one MSC batch is illustrated in the Fig. 3a. The wells include technological triplicates of five MSC dilutions (625, 1,250, 2,500, 5,000 and 10,000 cells per well) and five controls (MSC only, not-labeled and CFSE-labeled T 
cells in resting conditions, and not labeled and CFSE-labeled T cells in activating conditions). T cell proliferation is evaluated by CFSE dilution after three days of culture using flow-cytometry analysis, and the results are interpreted in relation to the negative (resting labeled $\mathrm{T}$ cells) and positive (labeled $\mathrm{T}$ cells cultured in activating conditions) controls. Gating strategy is illustrated in Fig. 3b, for the negative and positive controls. The population to be analyzed is selected by eliminating the debris (based on FSC and SSC scatter plot) and doublets (based on FSC-A vs. FSC-H), as well as dead cells (Propidium iodidepositive cells). The CFSE dilution is evaluated in the population of FITC ${ }^{\text {pos}} /$ Propidium iodide $^{\text {neg }}$ cells on the FITC histogram, where multiple fluorescence peaks are visible in the positive control, as compared to a single fluorescence peak in the negative control. One can note that the peak of not-activated T cells (the negative control) is one order of magnitude lower than the peak of labeled $T$ cells at time $t=0$, with a median fluorescence intensity of $7 \times 10^{\wedge} 5$ after culture $v s 7 \times 10^{\wedge} 6$ before culture (Figs. $2 \mathrm{~b}$ and $3 \mathrm{~b}$ ). The phase contrast microscopy of cells in co-culture is illustrated in Fig. 3c. The data show that irradiated MSC maintained the viability and appeared as a confluent monolayer of cells after four days in culture. Furthermore, the proliferation of $T$ cells is clearly visible in activating conditions ( $T$ cells + beads), in comparison to not-activated T cells ( $T$ cells only) and activated T cells in the presence of MSC (MSC $+T$ cells + beads).

\section{A dose-dependent inhibitory effect of MSC on T cell proliferation.}

The proliferation is being reported as proliferation index, which can be calculated using ModFit LT 5.0 software (Fig. 4). Usually, lymphocyte proliferation can be monitored by flow cytometry for up to eight divisions before CFSE fluorescence is decreased to the background fluorescence of unlabeled cells (14). The parent population is set based on the peak of the negative control ("T cells" sample in Fig. 4). This peak gradually diminishes as the cells proliferate in culture; lower the parent population peak, higher the proliferation rate. The results show a dose-dependent inhibition of T cell proliferation induced by the presence of MSC. By using increasing MSC to T cells ratios, a large range of inhibition can be achieved, which covers the whole interval between the positive and negative controls. Thus, total inhibition of T cell proliferation was obtained when MSC were present in a ratio of around 1:10 (MSC: T cells), while minimal inhibition was achieved with a 1: 160 (MSC: T cell) ratio. This data demonstrates strong immunosuppressive effects of MSC on activated T cells in culture.

\section{MSC irradiated versus non-irradiated.}

The mitotic inactivation of MSC is crucial in order to obtain a reliable dose-dependent effect on $\mathrm{T}$ cell proliferation. Figure 5a illustrates the loss of the dose dependence of the MSC inhibition when 
proliferating MSC are used in co-culture. Expectedly, higher inhibition was achieved with actively proliferating MSC, as compared to mitotically inactive MSC. The difference was more pronounced for lower doses of MSC as compared to higher doses, an effect that can be explained by the alteration of T cell: MSC ratio during co-culture, as a direct consequence of increasing the MSC number. In our hands, the mouse bone marrow-derived MSC have a population doubling time of around 50 hours, between 6th -14 th passages, which would allow for almost 2 population doublings during a 4-day culture period.

\section{Applications of the methods.}

As MSC have strong and dose-dependent immunosuppressive effects of T cells in vitro, differences between MSC batches, passages or conditions can be evaluated within the same experiment, by direct comparisons. Our data showed that no differences in the immunosuppressive properties existed between MSC at passages 7 and 11 of the same batch (Fig. 5b). Comparisons between different experiments can also be obtained by reporting the results as percentage of maximum proliferation. The normalization is required when data from different experiments are to be compared, due to the variation of PI value of activated $T$ cells between different experiments, which also affect the percentage value of resting $T$ cell proliferation in each individual experiment. For such comparisons, the maximum proliferation in each individual experiment was obtained by subtracting the PI value of resting T cells from the PI value of activated T cells. Figure $5 \mathrm{c}$ illustrates the comparative analysis of the immunosuppressive properties of four MSC batches obtained in separate experiments. All batches have inhibitory effects of T cell proliferation in culture. One batch (batch 4 at passage 10) was analyzed two times in independent experiments (performed in different days) and the results confirmed the reliability of the method (Fig. 5c). Comparisons between cells evaluated in different experiments can be done by plotting the $T$ cell proliferation against the absolute number of MSC in co-culture (represented as log2 scale) and calculating the area under the curve (AUC). Thus, our results revealed differences in the immunosuppressive capacity of various MSC batches, with batch 4 showing stronger inhibitory effects on $T$ cells than the other batches.

\section{Discussions}

\section{Starting material.}

MSC are most frequently isolated from bone marrow or adipose tissue. They are selected based on the adherence to the culture substrate and, usually, require several passages for depletion of contaminating hematopoietic cells, if no other methods of selection are chosen. Over the years, several protocols for mouse bone marrow MSC isolation were evaluated in our laboratory $(12,15,16)$. Particularly, in the case of $\mathrm{C} 57 \mathrm{BI} / 6$ mouse strain, which is reportedly the most popular mouse strain used in scientific research, purification of MSC culture takes several weeks (17). However, good results were obtained with a simple 
method, which started from plating of the whole bone marrow aspirate (under culture conditions with $5 \%$ oxygen atmosphere, when available) and purification of MSC by a couple of passages, at weekly or longer intervals. Particularly for $\mathrm{C} 57 \mathrm{BI} / 6$ mice, we observed that the depletion of $\mathrm{CD} 45^{\text {pos }}$ cells took longer than other strains and usually the first passage at which the culture was completely free of CD45 cells was passage 6 or 7 . Once established in culture, the cells maintained their properties for at least 10 passages after the complete removal of hematopoietic cells.

In contrast to mouse MSC, human MSC can be obtained more rapidly from bone marrow (11). The same is true for rat bone marrow, as well as from adipose tissue (of either species, human, mouse, rat)(18). When these sources are used, MSC are readily obtained from the first passages, however, they become senescent and stop proliferating earlier (after around 4 passages for rat bone marrow and 6 passages for adipose tissue-derived cells) $(19,20)$.

\section{Comparison with other methods.}

To evaluate the immunosuppressive capacity of MSC, it is important to have a standardized procedure that can follow lymphocyte proliferation with minimal disruption to cell viability. The ${ }^{3} \mathrm{H}$-thymydine assay has become a gold-standard protocol to monitor rates of DNA synthesis and cell proliferation in mixed lymphocyte reaction experiments performed in vitro (21-23). However, flow cytometry methods available today offer similar specificity and sensitivity with extra information in addition to the overall proliferative response (24-26). The intracellular fluorescent dye, CFSE, has been found to be particularly effective at monitoring cell proliferation in vitro, as it is stably incorporated within cells and its fluorescence is sequentially splitting to half with each cell division (24). Previous reports used CFSE-labeled lymphocytes to measure proliferation in the presence of proliferative MSC (27-31). The advantage of the protocol described in this study is given by the irradiation of MSC and the use of multiple doses, which allowed a more accurate dose-dependent estimation of the inhibitory effect. The maximum dose of MSC used in our study was calculated so that to form a confluent layer of MSC, over which an unnecessary cell crowding would be favored with negative effects on nutrient availability during the 3-day co-culture. Previous reports showed that heat-inactivated MSC did not suppress T cell proliferation (32). In contrast, our paper showed that co-culture of anti-CD3/CD28-activated T cells with irradiation-inactivated MSC strongly inhibited T cell proliferation in a dose dependent manner.

\section{Controls.}

It is important to have CFSE-labeled unstimulated T cells as a control, as they provide the fluorescence median of non-dividing lymphocytes. This control will also help identifying the starting fluorescence peak (the parent population) in proliferating cells. Using the CFSE-labeled T cells at time 0 to set the initial fluorescence level is not adequate, as the cells are extremely fluorescent within the first hours after staining and detection problems appear. Besides, the cells partially lose incorporated CFSE during the 3- 
day culture and consequently the CFSE-labeled resting T cells sample after 3 days in culture is the appropriate negative control for proliferation. Another important control is represented by unlabeled stimulated cells, in order to accurately set the autofluorescence level, knowing that the autofluorescence of proliferating lymphocytes is higher than that of resting lymphocytes.

\section{Conclusions}

This paper presents a standardized protocol to assess the immunosuppressive effect of MSC in vitro. The validation of the immunomodulatory capacity of MSC could be added to the characterization of the cells before being used in various MSC-based approaches to treat immunological diseases. Our results showed that MSC have a dose-dependent inhibitory effect on activated T cell proliferation. The immunosuppressive properties of MSC vary between batches, but not between different passages of the same batch.

\section{Abbreviations}

MSC - mesenchymal stem/stromal cells

CFSE - Carboxy-fluorescein succinimidyl ester

PI - proliferation Index

AUC - Area under the Curve

\section{Declarations}

\section{Ethics approval and consent to participate}

Not applicable.

\section{Consent for publication}

Not applicable.

\section{Availability of data and materials}

The data that support the findings of this study are available from the corresponding author upon reasonable request.

\section{Competing interests}


The authors declare that they have no competing interests.

\section{Funding}

This work was supported by a project cofinanced by the European Regional Development Fund through the Competitiveness Operational Program 2014-2020 (POC-A.1-A.1.1.4-E-2015, ID: P 37 668, acronym DIABETER) and Romanian Ministry of Education (PN-III-P1-1.1-PD-2016-1903, contract no 133PD/2018).

\section{Authors' contributions}

$\mathrm{CIM}$ and BMP performed experiments, analyzed the data and wrote the manuscript. AB designed the work, analyzed and interpreted the data, wrote the manuscript, and gave the final approval of the manuscript.

\section{Acknowledgements}

The authors would like to thank Dr. Nadir Askenasy and Dr. Maya Simionescu for their valuable advice on the data analysis and interpretation.

\section{References}

1. Bianco P, Robey PG, Simmons PJ. Mesenchymal stem cells: revisiting history, concepts, and assays. Cell Stem Cell. 2008;2(4):313-9.

2. Neuhuber B, Swanger SA, Howard L, Mackay A, Fischer I. Effects of plating density and culture time on bone marrow stromal cell characteristics. Exp Hematol. 2008;36(9):1176-85.

3. Kern S, Eichler H, Stoeve J, Kluter H, Bieback K. Comparative analysis of mesenchymal stem cells from bone marrow, umbilical cord blood, or adipose tissue. Stem Cells. 2006;24(5):1294-301.

4. da Silva Meirelles L, Caplan Al, Nardi NB. In search of the in vivo identity of mesenchymal stem cells. Stem Cells. 2008;26(9):2287-99.

5. Dominici M, Le Blanc K, Mueller I, Slaper-Cortenbach I, Marini F, Krause D, et al. Minimal criteria for defining multipotent mesenchymal stromal cells. The International Society for Cellular Therapy position statement. Cytotherapy. 2006;8(4):315-7.

6. Ma S, Xie N, Li W, Yuan B, Shi Y, Wang Y. Immunobiology of mesenchymal stem cells. Cell Death Differ. 2014;21(2):216-25.

7. Ren G, Zhang L, Zhao X, Xu G, Zhang Y, Roberts Al, et al. Mesenchymal stem cell-mediated immunosuppression occurs via concerted action of chemokines and nitric oxide. Cell Stem Cell. 2008;2(2):141-50. 
8. Weiss ARR, Dahlke MH. Immunomodulation by Mesenchymal Stem Cells (MSCs): Mechanisms of Action of Living, Apoptotic, and Dead MSCs. Front Immunol. 2019;10:1191.

9. Dazzi F, Ramasamy R, Glennie S, Jones SP, Roberts I. The role of mesenchymal stem cells in haemopoiesis. Blood Rev. 2006;20(3):161-71.

10. Keating A. Mesenchymal stromal cells. Curr Opin Hematol. 2006;13(6):419-25.

11. Krampera M, Galipeau J, Shi Y, Tarte K, Sensebe L, Therapy MSCCotISfC. Immunological characterization of multipotent mesenchymal stromal cells-The International Society for Cellular Therapy (ISCT) working proposal. Cytotherapy. 2013;15(9):1054-61.

12. Rosca AM, Burlacu A. Effect of 5-azacytidine: evidence for alteration of the multipotent ability of mesenchymal stem cells. Stem Cells Dev. 2011;20(7):1213-21.

13. Hathcock KS. T cell enrichment by nonadherence to nylon. Curr Protoc Immunol. 2001;Chap. 3:Unit 3 2.

14. Quah BJ, Warren HS, Parish CR. Monitoring lymphocyte proliferation in vitro and in vivo with the intracellular fluorescent dye carboxyfluorescein diacetate succinimidyl ester. Nat Protoc. 2007;2(9):2049-56.

15. Soleimani M, Nadri S. A protocol for isolation and culture of mesenchymal stem cells from mouse bone marrow. Nat Protoc. 2009;4(1):102-6.

16. Boregowda SV, Krishnappa V, Phinney DG. Isolation of Mouse Bone Marrow Mesenchymal Stem Cells. Methods Mol Biol. 2016;1416:205-23.

17. Peister A, Mellad JA, Larson BL, Hall BM, Gibson LF, Prockop DJ. Adult stem cells from bone marrow (MSCs) isolated from different strains of inbred mice vary in surface epitopes, rates of proliferation, and differentiation potential. Blood. 2004;103(5):1662-8.

18. Phinney DG, Kopen G, Isaacson RL, Prockop DJ. Plastic adherent stromal cells from the bone marrow of commonly used strains of inbred mice: variations in yield, growth, and differentiation. $J$ Cell Biochem. 1999;72(4):570-85.

19. Burlacu A, Rosca AM, Maniu H, Titorencu I, Dragan E, Jinga V, et al. Promoting effect of 5-azacytidine on the myogenic differentiation of bone marrow stromal cells. Eur J Cell Biol. 2008;87(3):173-84.

20. Preda MB, Ronningen T, Burlacu A, Simionescu M, Moskaug JO, Valen G. Remote transplantation of mesenchymal stem cells protects the heart against ischemia-reperfusion injury. Stem Cells. 2014;32(8):2123-34.

21. Krampera M, Glennie S, Dyson J, Scott D, Laylor R, Simpson E, et al. Bone marrow mesenchymal stem cells inhibit the response of naive and memory antigen-specific $T$ cells to their cognate peptide. Blood. 2003;101(9):3722-9.

22. Krampera M, Cosmi L, Angeli R, Pasini A, Liotta F, Andreini A, et al. Role for interferon-gamma in the immunomodulatory activity of human bone marrow mesenchymal stem cells. Stem Cells. 2006;24(2):386-98. 
23. Goodell V, dela Rosa C, Slota M, MacLeod B, Disis ML. Sensitivity and specificity of tritiated thymidine incorporation and ELISPOT assays in identifying antigen specific T cell immune responses. BMC Immunol. 2007;8:21.

24. Quah BJ, Parish CR. New and improved methods for measuring lymphocyte proliferation in vitro and in vivo using CFSE-like fluorescent dyes. J Immunol Methods. 2012;379(1-2):1-14.

25. Lastovicka J, Rataj M, Bartunkova J. Assessment of lymphocyte proliferation for diagnostic purpose: Comparison of CFSE staining, Ki-67 expression and (3)H-thymidine incorporation. Hum Immunol. 2016;77(12):1215-22.

26. Ten Brinke A, Marek-Trzonkowska N, Mansilla MJ, Turksma AW, Piekarska K, Iwaszkiewicz-Grzes D, et al. Monitoring T-Cell Responses in Translational Studies: Optimization of Dye-Based Proliferation Assay for Evaluation of Antigen-Specific Responses. Front Immunol. 2017;8:1870.

27. van den Akker F, Vrijsen KR, Deddens JC, Buikema JW, Mokry M, van Laake LW, et al. Suppression of $T$ cells by mesenchymal and cardiac progenitor cells is partly mediated via extracellular vesicles. Heliyon. 2018;4(6):e00642.

28. Ren G, Su J, Zhang L, Zhao X, Ling W, L'Huillie A, et al. Species variation in the mechanisms of mesenchymal stem cell-mediated immunosuppression. Stem Cells. 2009;27(8):1954-62.

29. Zinocker S, Vaage JT. Rat mesenchymal stromal cells inhibit T cell proliferation but not cytokine production through inducible nitric oxide synthase. Front Immunol. 2012;3:62.

30. Anderson P, Carrillo-Galvez AB, Garcia-Perez A, Cobo M, Martin F. CD105 (endoglin)-negative murine mesenchymal stromal cells define a new multipotent subpopulation with distinct differentiation and immunomodulatory capacities. PLoS One. 2013;8(10):e76979.

31. Nauta AJ, Westerhuis G, Kruisselbrink AB, Lurvink EG, Willemze R, Fibbe WE. Donor-derived mesenchymal stem cells are immunogenic in an allogeneic host and stimulate donor graft rejection in a nonmyeloablative setting. Blood. 2006;108(6):2114-20.

32. Luk F, de Witte SF, Korevaar SS, Roemeling-van Rhijn M, Franquesa M, Strini T, et al. Inactivated Mesenchymal Stem Cells Maintain Immunomodulatory Capacity. Stem Cells Dev. 2016;25(18):1342-54.

\section{Figures}



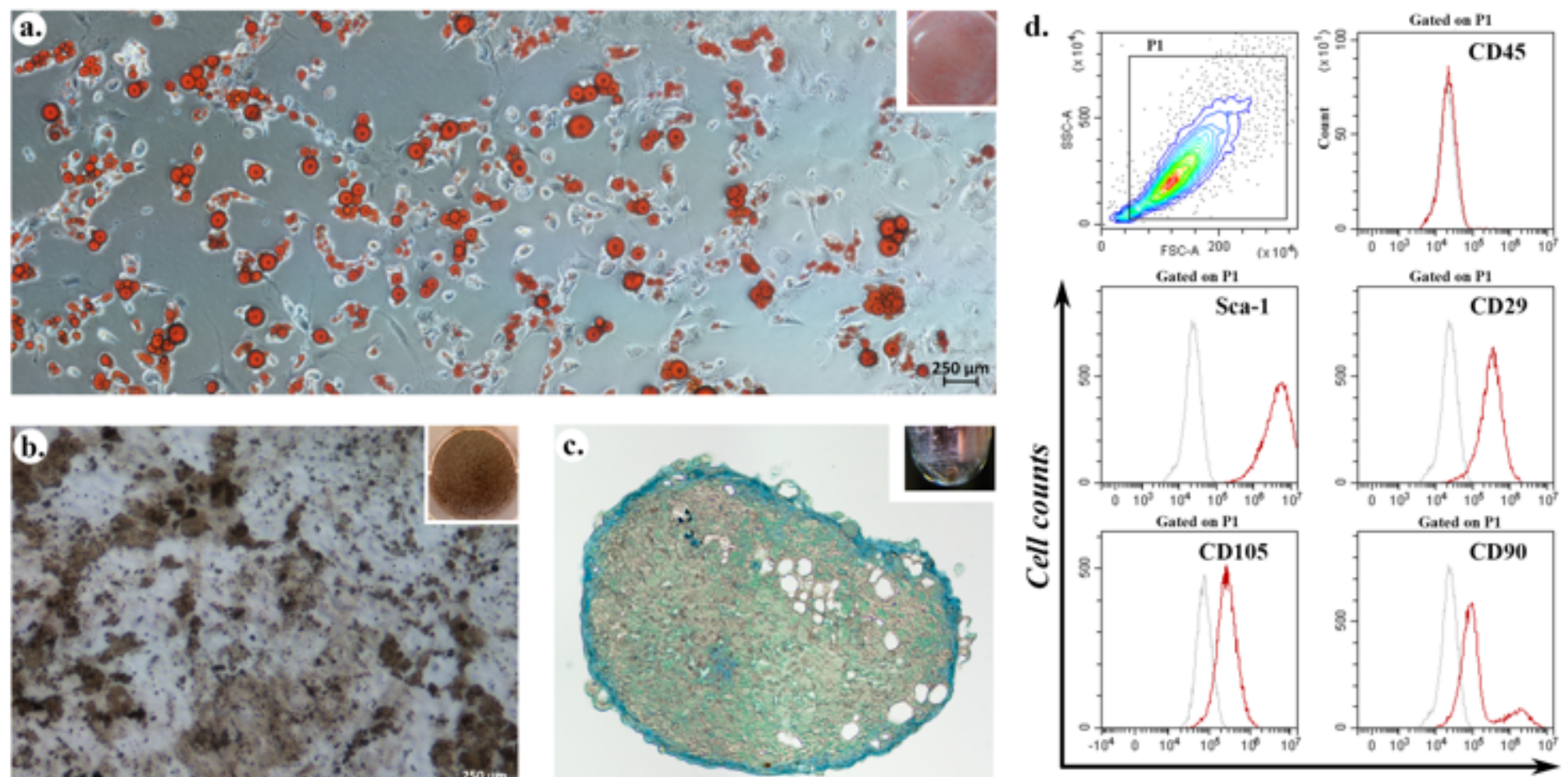

Fluorescence intensity

Figure 1

MSC characterization in accordance to International Society for Cellular Therapy. Oil red (a), von Kossa (b) and Alcian Blue (c) staining demonstrating the capacity of MSC to differentiate in adipocytes, osteoblasts and chondroblasts, respectively, when cultured in appropriate differentiation media for three weeks. Insets show the aspect of whole samples analyzed, from which a representative field was selected for illustration. d) Phenotypic characterization of MSC by flow cytometry, illustrating the lack of CD45 expression and the expression of Sca-1, CD29, CD105, and CD90.

a.
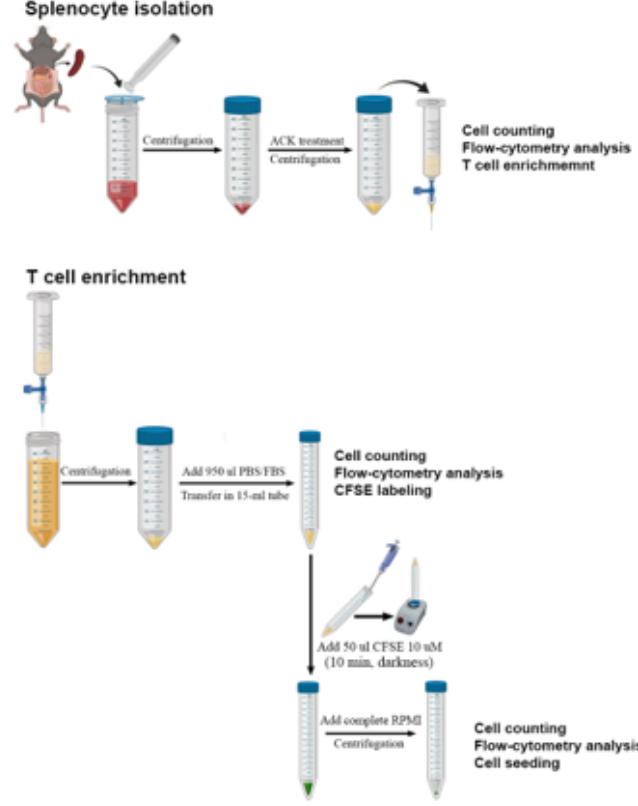

b.
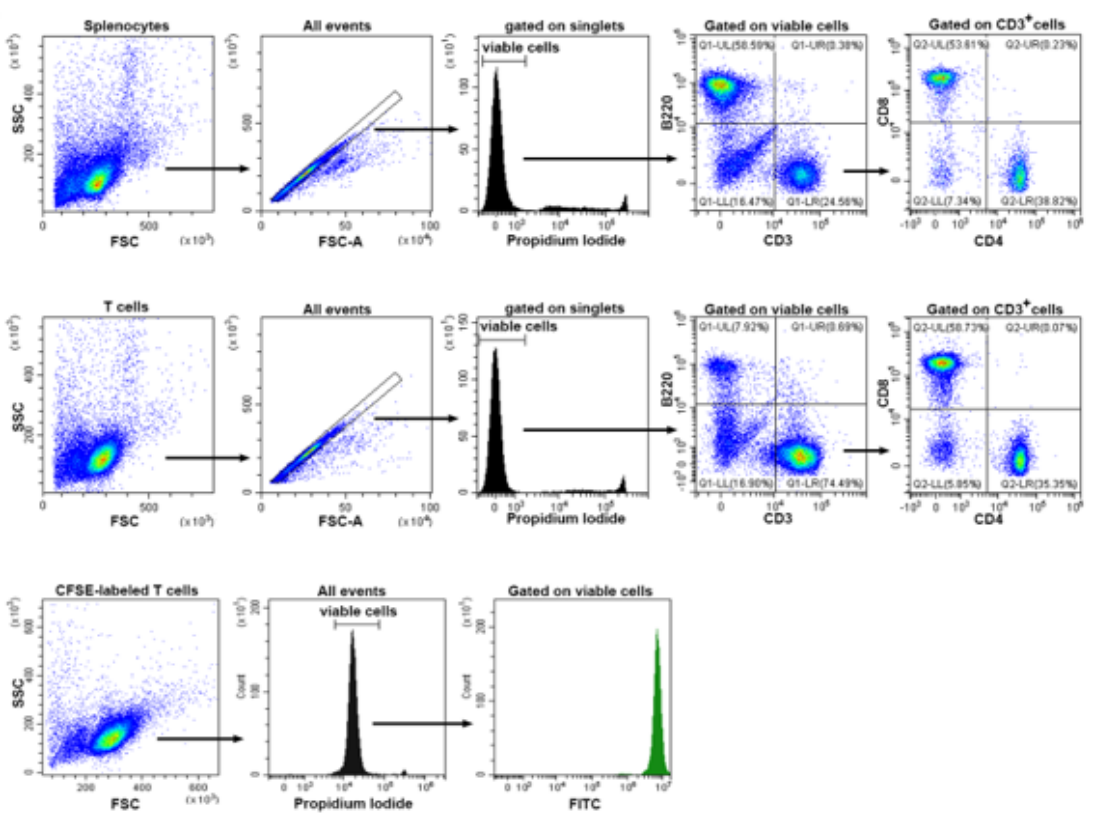
Figure 2

Schematic design of the main steps for T cell isolation and CFSE-labeling. a) Isolation of mouse splenocytes and T cell enrichment on nylon wool column. The critical step of CFSE staining of T cells is also shown. b) Flow-cytometry characterization of splenocytes (upper line), $\mathrm{T}$ cells (middle line) and CFSE-labeled T cells (lower line). Note the depletion in B220pos cells in T cell enriched population, as compared to whole splenocyte population ( $~ 8 \%$ B cells after enrichment, as compared to $\sim 60 \%$ in the initial population) and the relatively unchanged CD8:CD4 ratio after enrichment. Also note the sharp fluorescent peak of T cell population obtained after CFSE staining.

a.

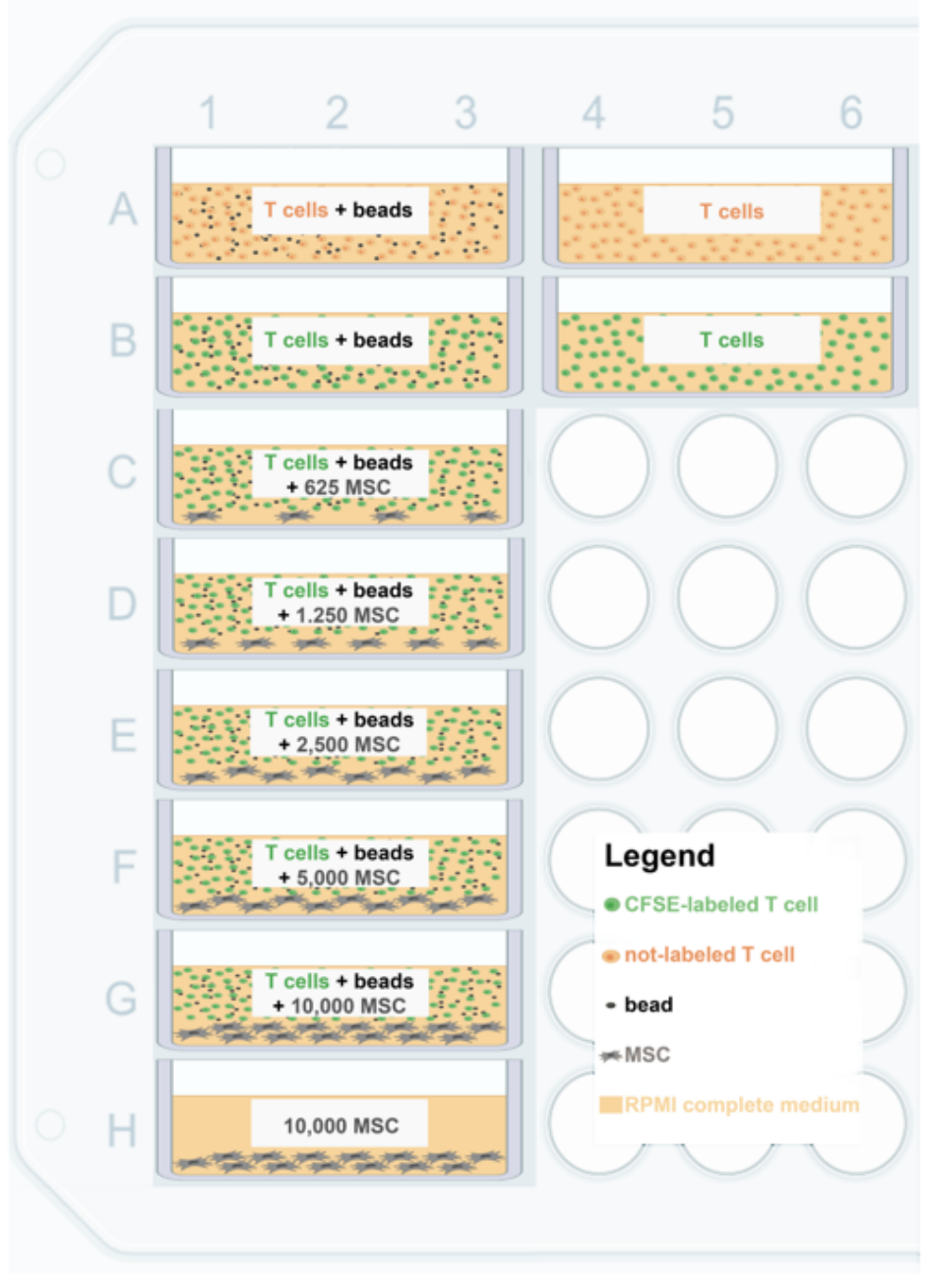

b.
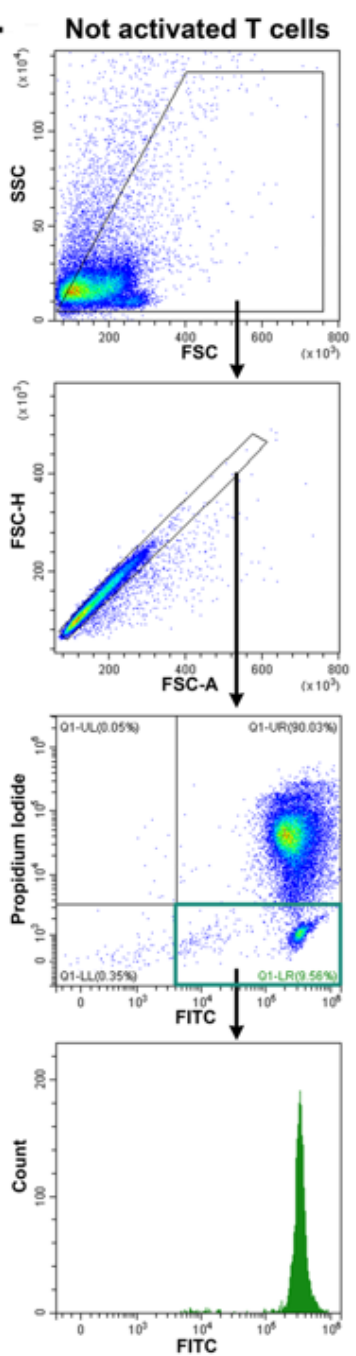
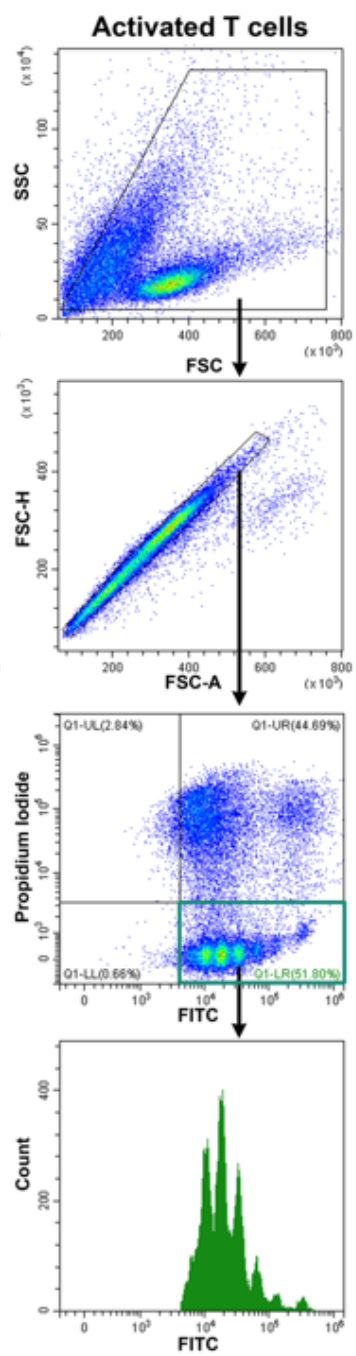

c. MSC only

T cells only

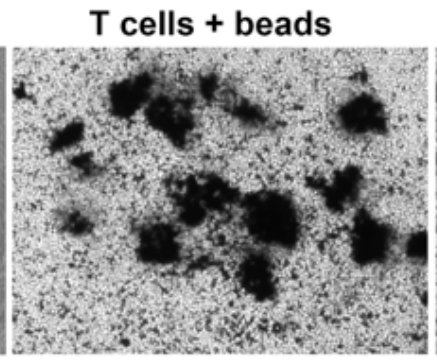

MSC + T cells + beads

as 


\section{Figure 3}

a) The 96-well plate template for experimental design. Thirty wells are required for testing one MSC batch, of which 12 wells serve as controls (the first two lines of the plate). Each additional MSC batch will require another 18 wells, which can be aligned as triplicate columns after the first batch. A total of four different batches can be analyzed in one 96-well plate. b) Flow cytometry analysis of the T cell population in the negative and positive controls, at the end of three-day co-culture experiment. The gating strategy consists of removal of debris, doublets and dead cells and analysis of the viable cells on the CFSE histogram. Multiple fluorescent peaks are distinguished in the positive control (activated T cells), as compared to the single peak in the negative control (not activated T cells). c) Phase-contrast microscopy images of irradiated MSC, resting T cells and activated T cells in culture (in the presence or absence of MSC). 

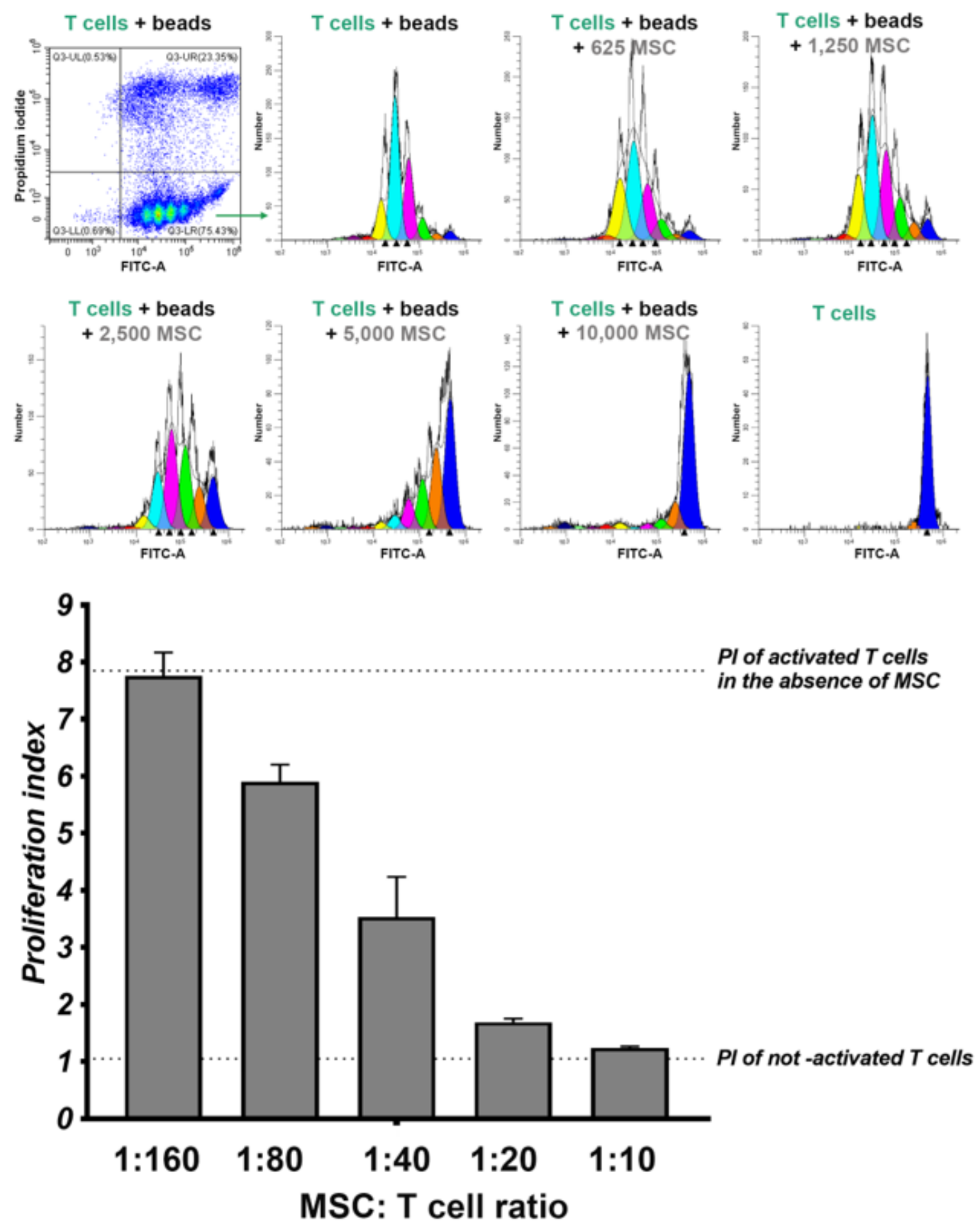

Figure 4

T cell proliferation in the presence of MSC. Histograms illustrate the CFSE fluorescent peaks of T cell samples activated in the presence of increasing amount of MSC. Note the single fluorescent peak in resting $T$ cells, representing the parent population. Also note the diminishing of the parent population peak with the increasing number of MSC in the culture, concomitant with the increasing in the magnitude of the low-fluorescent peaks, as a result of dye dilution with each cell proliferation. The diagram shows 
the decrease of the proliferation index $(\mathrm{PI})$ of activated T cells in the presence of MSC in a dosedependent manner.

a.

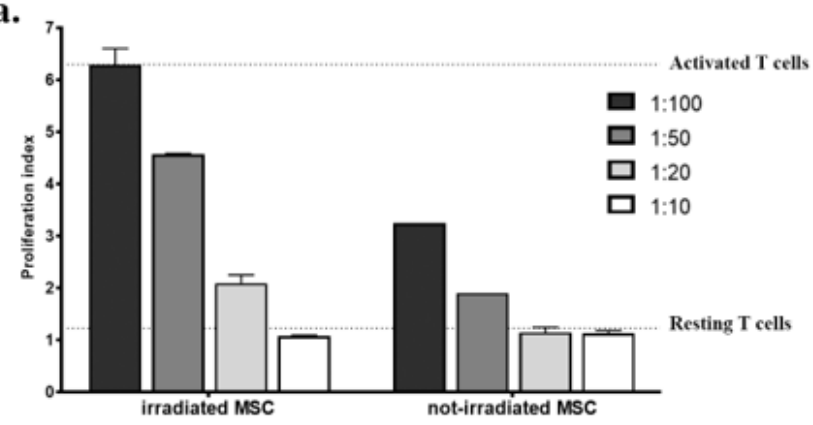

b.

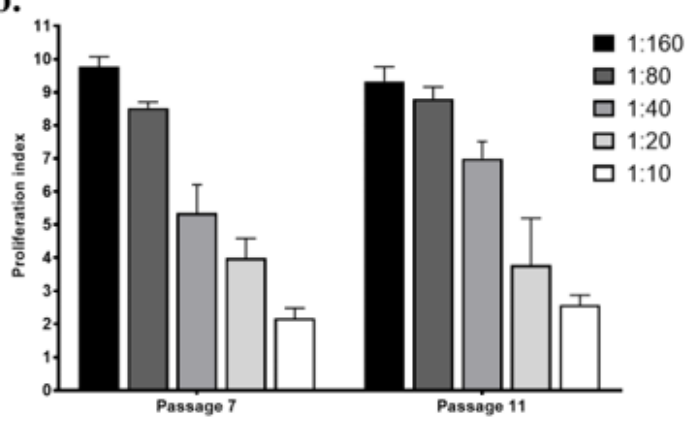

c.
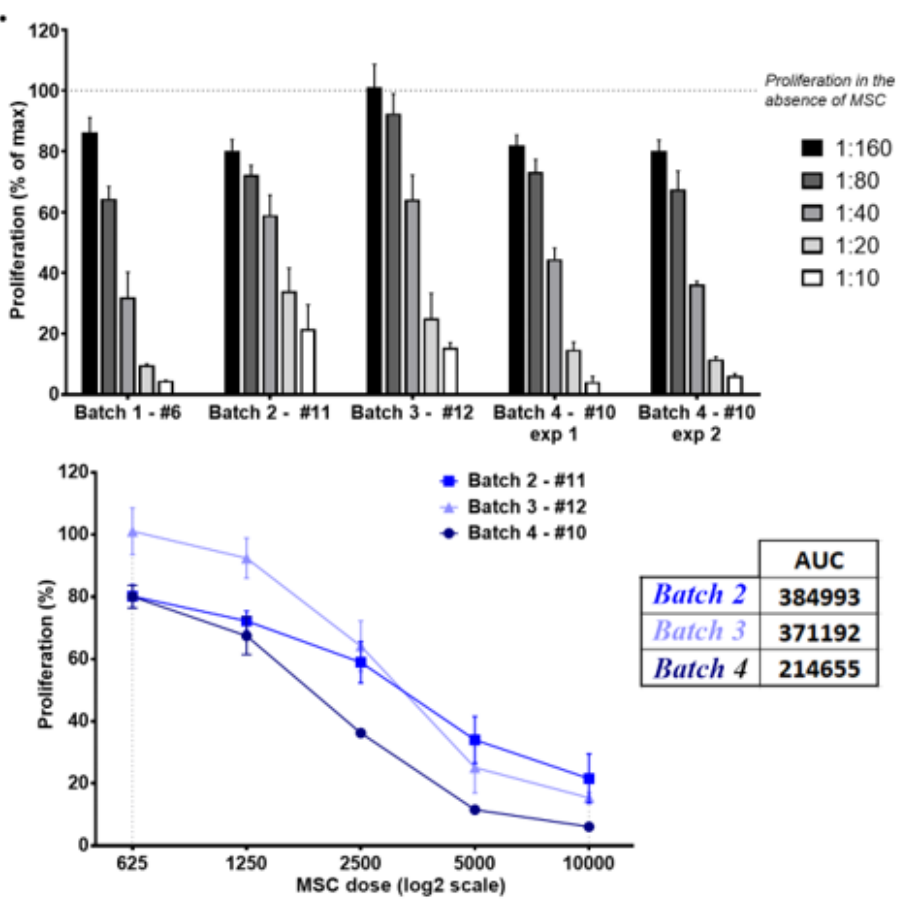

Figure 5

Dose-dependent inhibition of T cell proliferation by MSC. a) The comparative illustration of the effect of actively proliferating and irradiated MSC on T cell proliferation. Note the loss of the dose-dependent effect of proliferative MSC on the inhibition of T cell proliferation. b) Comparative analysis of the immunosuppressive effect of a batch of MSC at a low and a higher passage. Note that these MSC properties are not modified with increasing the passage number. The experiment represents one representative experiment from at least 6 experiments performed with different MSC batches. c) Comparative analysis of the immunosuppressive properties of 4 batches of MSC measured in separate experiments. The comparison was done by reporting the proliferation as percentage of maximum proliferation obtained in individual experiments. By plotting the proliferation against the dose of MSC, the area under curve can be calculated, which makes possible the comparison between the immunosuppressive properties of different batches. Lower the AUC, higher the immunosuppressive effects. 